\section{Spijker y Gumà confirman que durante la crisis económica en España hubo una mejora de indicadores de salud}

\author{
Spijker and Gumà confirm that \\ during the economic crisis there \\ was an improvement in health \\ indicators in Spain
}

José A. Tapia Granados ${ }^{1}$

${ }^{1}$ Médico. Doctor en Economía. Profesor titular, Department of Politics, Drexel University, Filadelfia, EEUU. $\triangle$ iD

doi: 10.18294/sc.2018.1987

Discusión sobre: Spijker J, Gumà J. El efecto de la crisis económica sobre la salud en España según el nivel educativo y la relación con la actividad: ¿importa también la duración de la crisis? Salud Colectiva. 2018;14(4):655670. doi: 10.18294/sc.2018.1297

Jeroen Spijker y Jordi Gumà ${ }^{(1)}$ analizan los cambios de un indicador subjetivo de salud en tres encuestas que se hicieron en 2006, 2010 y 2014 en España; según Eurostat, la tasa de desempleo alcanzó un mínimo de $6,1 \%$ en marzo de 2007 y durante la crisis subió hasta alcanzar un máximo de 26,3\% en julio de 2013. Spiker y Gumà comparan tres situaciones: 2006, cuando la expansión económica, en gran parte debida al sector de la construcción ("el ladrillazo"), se acercaba a su final; 2010, cuando la recesión estaba en pleno desarrollo y el desempleo llegaba al $20 \%$; y 2014, cuando la crisis comenzaba a remitir. Las encuestas que Spijker y Gumà usan son parte de la European Union Statistics on Income and Living Conditions (EU-SILC), en las que la unidad muestral fueron hogares seleccionados probabilísticamente para que las muestras fueran representativas de la población española. Sin embargo, Spijker y Gumà excluyeron del análisis los nacidos fuera del territorio español, que forman una minoría rápidamente creciente de la población con nacionalidad española.

Spijker y Gumà comienzan afirmando que todavía no se ha establecido de manera clara "la relación entre un contexto económico adverso y la salud", y especulan que si en una crisis se compromete la sanidad pública puede afectar la salud de quienes precisan atención y no pueden recibir las mismas prestaciones que antes. En realidad, son múltiples los estudios que muestran una mejora de la salud en las recesiones, por ejemplo, en Argentina $^{(2)}$, y atribuir una influencia intensa e inmediata de los servicios de salud sobre la salud poblacional es algo que los epidemiólogos ven en general con escepticismo ${ }^{(3)}$. De hecho, durante las recesiones a menudo empeoran los servicios de salud a la vez que baja la mortalidad ${ }^{(4)}$, lo que no parece compatible con una influencia decisiva de la atención médica en la salud poblacional.

Los resultados de Spijker y Gumà son, a mi juicio, uno más de los estudios que confirman que en economías de mercado asentadas, los periodos de recesión coinciden con mejoras de la salud. Si eso es particularmente obvio cuando la salud se evalúa por la mortalidad $^{(5)}$, que aumenta en las expansiones y disminuye en las crisis, estudios como este y otros son ejemplos de que los indicadores de morbilidad o bienestar también tienden a mejorar durante las crisis $^{(6,7)}$ a pesar de que la salud mental parece deteriorarse y en años de recesión, en general, aumentan los suicidios. Lamentablemente, a pesar de que todo esto se ha hallado en repetidos estudios, muchos epidemiólogos continúan tomando los estudios de Harvey Brenner y Ralph Catalano como si probaran lo contrario. Brenner con métodos obscuros que no se han podido replicar y que han recibido muchas críticas, supuestamente demostró que las crisis hacían aumentar la mortalidad con un desfase, cuya duración nunca aclaró. Catalano tras mantener posiciones similares a Brenner, supuestamente demostró que las recesiones no tienen ningún efecto observable sobre la mortalidad, lo que ha sido refutado ${ }^{(8)}$. Muchos epidemiólogos 
ponen demasiado énfasis en que el nivel de ingreso y la situación laboral son determinantes clave del nivel de salud. Ciertamente hay un gradiente de mortalidad por nivel de ingreso: las personas de menos ingresos tienen más riesgo de muerte y en general los desempleados tienen peor salud que los empleados. Pero otros factores como la dieta, la actividad física, el estado civil, el nivel de integración social y las exposiciones en el ambiente laboral también influyen en gran medida sobre el riesgo de muerte. Spijker y Gumà no mencionan la influencia sobre la salud de las condiciones de trabajo a las que una gran mayoría de la población adulta suele estar expuesta ocho horas diarias cinco días a la semana y muchas veces bastante más. La semana laboral de 40 horas y el mes de vacaciones anuales eran "lo normal" en España en los años ochenta y noventa, pero la precarización y las sucesivas reformas laborales (pactadas con los sindicatos o impuestas pese a su resistencia) llevaron a horarios más extensos y flexibles que, de seguro, se extendieron para responder a las demandas del mercado en los años del ladrillazo. Lamentablemente, los epidemiólogos han prestado poca atención a las condiciones de trabajo, que claramente dependen de la fase del ciclo económico ya que, por ejemplo, las horas de trabajo y la frecuencia de traumatismos y lesiones aumentan en las expansiones y disminuyen en las recesiones.

Lo más significativo de los resultados de Spijker y Gumà es que la autopercepción de mala salud disminuye en todos los grupos de varones o mujeres en la primera fase de la crisis, entre 2006 y 2010, y en casi todos los grupos -excepto unos pocos en los que aumenta muy débilmente- en la segunda fase de la crisis, entre 2010 y 2014. Esto no es compatible con un efecto nocivo de la crisis sobre la salud, pero sí lo es con un efecto beneficioso, que Spijker y Gumà son reacios a admitir.

Spijker y Gumà presentan como hipótesis de investigación que las diferencias de nivel de salud entre personas de mayor o menor nivel educativo o entre empleados y desempleados aumentaron como consecuencia de la crisis. "En caso afirmativo, eso permitiría entender por qué a nivel poblacional, los efectos de la crisis sobre la salud no resultan visibles, ya que una parte de la población [...] se ve afectada, y otra parte no"(1). Sin embargo, al discutir los resultados del estudio no se indica si esa hipótesis se confirma o no.

En 2006, el 38,3\% de los varones desempleados consideraron no tener buena salud mientras que la proporción correspondiente fue del 20,1\% en empleados; en 2014 las proporciones correspondientes fueron del $24,3 \%$ y el $12,7 \%$. Así, suponiendo que la crisis empezó en 2007, tras siete años de crisis, la proporción de quienes dicen tener mala salud se ha reducido casi a la mitad en ambos grupos y la razón de proporciones desempleados/empleados que, en 2006, era casi del doble $(38,3 / 20,1=1,9)$ se mantiene en $2014(24,3 / 12,7=1,9)$. Entre mujeres desempleadas y empleadas, la razón de proporciones en 2006 fue $29,6 / 21,1=1,4$ y, en 2014, $28,4 / 15,5=1,8$, de forma que hubo también mejora en ambos grupos, pero la diferencia aumentó. Entre personas con empleo, la proporción que manifestaba no tener buena salud en 2006 era del $20,1 \%$, en varones y del $21,1 \%$ mujeres, en 2014 era del $12,7 \%$ en varones y del $15,5 \%$ en mujeres. Así que hubo mejora en ambos grupos, pero en 2014 la mala salud autopercibida es más frecuente en mujeres mientras que, en 2006, lo era en varones.

También entre grupos definidos por nivel educativo, la diferencia de salud autopercibida parece aumentar ligeramente. Así la mala salud autopercibida en los varones con educación obligatoria comparados con los que tenían educación universitaria fue $28,1 / 15,8=1,8$ en 2006 y aumentó a $21,4 / 10,5=2,0$ en 2014. Estos datos sugieren un aumento de las diferencias de salud autopercibida entre grupos socioeconómicos y demográficos durante la crisis, lo que parece contradictorio con el descenso de la mortalidad durante la crisis, sobre todo en los grupos de peor situación socioeconómica ${ }^{(9)}$.

Tanto en varones como en mujeres clasificados, según dificultad financiera para llegar a fin de mes, la proporción de quienes no se sienten con buena salud disminuye entre 2006 y 2010 y de nuevo entre 2010 y 2014 con una excepción: la de los varones que 
dicen no tener dificultades financieras, en los que sorprendentemente aumenta la autopercepción de mala salud entre 2010 y 2014. Esto parece similar a lo observado por Kondo et al. ${ }^{(10)}$ en Japón, donde la crisis de los años noventa afectó significativamente la salud de gerentes y ejecutivos pero no la de personal subalterno y de ventas.

Quizá vale la pena señalar que la intensa reducción de la proporción con mala salud autopercibida entre varones desempleados entre $2006(38,3 \%)$ y $2010(25,2 \%)$ es fácil de explicar por un efecto de dilución. Probablemente, en 2006, entre los desempleados había muchos con mala salud que les dificultaba obtener un empleo a pesar de la fuerte demanda laboral revelada por una desocupación del 6\%, bajísima para estándares hispanos. En 2010, la tasa de desempleo se había triplicado e indudablemente entre los millones de desempleados la proporción con mala salud había bajado por el aflujo de muchos sanos que habían perdido su trabajo por cierres empresariales o despidos colectivos en expedientes de regulación de empleo.

Un aspecto destacable del trabajo es la enorme diferencia en el perfil de cambios entre varones y mujeres que ilustra la Figura 1 del artículo $^{(1)}$. En este, como en muchos otros estudios, se observa que los cambios macroeconómicos afectan mucho menos a las mujeres.

Este estudio brinda información muy valiosa sobre la evolución en España durante la crisis de un indicador de salud. Una limitación es que está basado en información obtenida de una muestra de menos del $0,1 \%$ de la población española. Una muestra de ese tipo, aunque se seleccione con todo cuidado para que sea representativa, siempre implica la posibilidad de sesgos importantes a los que, por ejemplo, no están expuestos los estudios de mortalidad basados en todas las defunciones registradas.

\section{REFERENCIAS BIBLIOGRÁFICAS}

1. Spijker J, Gumà J. El efecto de la crisis económica sobre la salud en España según el nivel educativo y la relación con la actividad: ¿importa también la duración de la crisis? Salud Colectiva. 2018;14(4):655-670. doi: 10.18294/ sc.2018.1297.
2. Abdala F, Geldstein RN, Mychaszula SM. Economic restructuring and mortality changes in Argentina: Is there any connection? En: Cornia GA, Paniccià R, (eds.). The mortality crisis in transitional economies. New York: Oxford University Press; 2000. p. 328-350.

3. McKinlay JB, McKinlay SM, Beaglehole R. Trends in death and disease and the contribution of medical measures. En: Freeman HE, Levine S, (eds.). Handbook of medical sociology. 2nd ed. Englewood Cliffs, NJ: Prentice Hall; 1989. p. $14-45$.

4. Tapia Granados JA, Rodriguez JM. Health, economic crisis, and austerity: A comparison of Greece, Finland and Iceland. Health Policy. 2015;119(7):941-953.

5. Tapia Granados JA. Macroeconomic effects on mortality: Issues, controversies, and directions for research. En: Scott R, Buchmann M, (eds.). Emerging trends in the social and behavioral sciences 2017. New York: John Wiley; 2017. p. 1-16.

6. Ruhm CJ. Healthy living in hard times. Journal of Health Economics. 2005;24(2):341-363.

7. Tapia Granados JA, Christine PJ, lonides EL, Carnethon MR, Diez Roux AV, et al. Cardiovascular risk factors, depression, and alcohol consumption during joblessness and during recessions in CARDIA young adults. American Journal of Epidemiology. 2018;187(11):2339-2345.

8. Tapia Granados JA, Ionides EL. Statistical evidence shows that mortality tends to fall during recessions: A rebuttal to Catalano and Bruckner. International Journal of Epidemiology. 2016;45(5):1683-1685.

9. Regidor E, Vallejo F, Tapia Granados JA, Viciana-Fernández FJ, Fuente L, Barrio G. Faster mortality decline in low socioeconomic groups during the economic crisis in Spain: A cohort study of 36 million people. Lancet. 2016;388:2642-2652.

10. Kondo N, Subramanian SV, Kawachi I, Takeda Y, Yamagata Z. Economic recession and health inequalities in Japan: Analysis with a national sample, 19862001. Journal of Epidemiology and Community Health. 2008;62(10):869-875.

\section{FORMA DE CITAR}

Tapia Granados JA. Spijker y Gumà confirman que durante la crisis económica en España hubo una mejora de indicadores de salud. Salud Colectiva. 2018;14(4):685687. doi: 10.18294/sc.2018.1987.

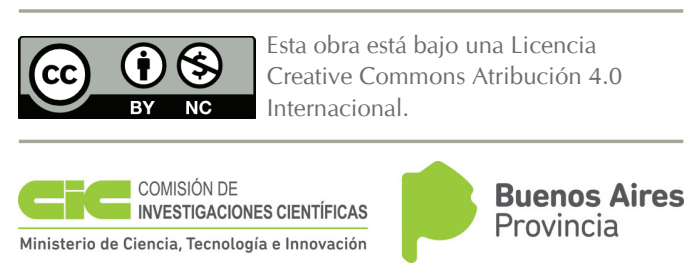

Este artículo fue traducido al español por Vanessa Di Cecco con fondos del Subsidio para Publicaciones de Divulgación Científica (Acta No 1459/17) de la Comisión de Investigaciones Científicas de la provincia de Buenos Aires. 